\title{
The conceptual advances of carcinogenic sequence model in high-grade serous ovarian cancer (Review)
}

\author{
HIROSHI KOBAYASHI, KANA IWAI, EMIKO NIIRO, SACHIKO MORIOKA, \\ YUKI YAMADA, KENJI OGAWA and NAOKI KAWAHARA
}

Department of Obstetrics and Gynecology, Nara Medical University, Kashihara, Nara 634-8522, Japan

Received July 5, 2017; Accepted July 20, 2017

DOI: 10.3892/br.2017.955

\begin{abstract}
The present review focuses on the current status of molecular pathology in high-grade serous cancer (HGSC) and preneoplastic conditions. This article reviews the English-language literature on HGSC, precursor, fallopian tubal epithelium, secretory cells, ciliated cells, secretory cell expansion, secretory cell outgrowth (SCOUT), p53 signature, serous tubal intraepithelial carcinoma (STIC), DNA damage and immunohistochemistry in an effort to identify the precursor-carcinoma sequence in HGSC. The majority of HGSC originates from the fimbriated end of the fallopian tube secretory epithelial cells, while the small part of this disease may develop from ovarian cortical inclusion cyst (CIC). A series of morphological changes from normal fallopian epithelium to preneoplastic to neoplastic lesions were concomitant with the multistep accumulation of molecular and genetic alterations. Recent studies provide a stepwise progression of fallopian tubal epithelium to precursor lesions to carcinoma, with the aid of a 'secretory cell-SCE-SCOUT-p53 signature-STIC-HGSC sequence' model. Immunohistochemical markers, including p53, STMN1, EZH2, CCNE1, Ki67 and $\gamma$-H2AX, were gradually increased during the SCOUT-p53 signature-STIC-HGSC sequence. Conversely, PAX2 expression was decreased during the early phase of SCOUT development. Potential genes and proteins are involved in the evolutionary trajectory of the precursor-cancer lineage model. In the present review we examined detailed aspects of the molecular changes involved in malignant transformation from fallopian tube epithelium to HGSC. A precursor condition originating in 'field cancerization' may gain a growth advantage, leading to HGSC.
\end{abstract}

Correspondence to: Dr Hiroshi Kobayashi, Department of Obstetrics and Gynecology, Nara Medical University, 840 Shijo-cho, Kashihara, Nara 634-8522, Japan

E-mail: hirokoba@naramed-u.ac.jp

Key words: high-grade serous cancer, fallopian tubal epithelium, serous tubal intraepithelial carcinoma, molecular pathology, stepwise progression

\section{Contents}
1. Introduction
2. Histopathology of precursor lesions
3. Molecular pathogenesis
4. Conclusion

\section{Introduction}

The lifetime risk of developing ovarian cancer is $16-59 \%$ for women with germline BRCA mutations and only $1.4 \%$ for women without germline mutations (1). Recent molecular genetics and morphologic characteristics revealed that ovarian cancer is divided into two categories, designated types 1 and 2 (2). Type 1 tumors exemplify the classically held view of a stepwise progression (adenoma-carcinoma sequence), which comprise endometriosis-associated ovarian cancer (EAOC), such as clear cell carcinoma and low-grade endometrioid carcinoma, as well as mucinous carcinoma and low-grade serous carcinoma. According to this model, it is generally accepted that the malignant lesion originates from pre-existing adenomas. Type 1 cancer develops through a particular sequence of somatic mutations or genomic alterations (ARID1A, PIK3CA, PTEN, KRAS, BRAF, CTNNB1, and PPP2R1A), with rare mutations in BRCA1, BRCA2, and TP53 (2). By contrast, type 2 tumors arise from the normal epithelium to precursor lesions and finally to high-grade serous and endometrioid carcinoma, malignant mixed mesodermal tumors (carcinosarcomas), and undifferentiated carcinoma.

High-grade serous cancers, including ovarian, tubal and pelvic cancers, are more aggressive and typically present in advanced stages, indicating that the evolutionary trajectory of type 2 cancer progression is rapid. High-grade serous cancer (HGSC) may develop from multiple extra-ovarian origins, including the fimbrial end of the fallopian tube and peritoneum (2). The small part of HGSC may originate from ovarian cortical inclusion cyst (CIC) (2). The majority of this disease may be the result of a stepwise process, from fallopian tubal epithelium to serous tubal intraepithelial carcinoma (STIC) to finally HGSC (1-13). However, we cannot rule out a 'parallel' evolution of synchronous precursor and cancer.

The present review focused on the current status of molecular pathology that initiates HGSC and preneoplastic 
conditions. The current review presents a stepwise model that incorporates both molecular alterations and the histopathology of precursor lesions.

General. The present study aimed to summarize the current status of molecular pathology in HGSC and preneoplastic conditions. A PubMed (http://www.ncbi.nlm.nih.gov/pubmed) search of the relevant literature published between 2005 and 2017 was performed. The search strategy included the combination of the following key words: high-grade serous cancer (HGSC), precursor, fallopian tubal epithelium, secretory cells, ciliated cells, secretory cell expansion (SCE), secretory cell outgrowth (SCOUT), p53 signature, STIC, DNA damage and immunohistochemistry in the titles or abstracts of articles. English-language publication search results from PubMed and references within the relevant articles were analyzed. To minimize selection bias, screening of the studies was independently performed by two of the co-authors (K.I. and E.I.) after agreeing on the selection criteria.

\section{Histopathology of precursor lesions}

We describe all possible origins of preneoplastic cellular alterations to HGSC, and discuss their pros and cons.

SCE. The first lesion is SCE. The oviduct comprises glands and a luminal epithelium which is composed of secretory cells and ciliated cells. The number of tubal secretory cells increases with age (4). An increase of secretory cells was observed in high-risk individuals and sporadic serous cancer cases (4). Although SCE may be a sensitive marker for early serous carcinogenesis in patients with coexisting HGSC, SCE is prevalent in both fimbria and ampulla tubal segments in fallopian tubal regions. Furthermore, animal experiments revealed an increase in the number of SCE and a decrease in the number of ciliated cells after hCG administration, indicating that SCE may serve a range of the multiple physiological roles of fallopian epithelial functions (14). Therefore, SCE is not directly linked to a precancerous lesion.

SCOUT. The second lesion is SCOUT, which contains a linear stretch of 30 or more fallopian epithelial cells of secretory type $(5,13,15)$. SCOUT was observed in $60-90 \%$ of the HGSC group and $20-70 \%$ of the normal control group $(3,12,15)$. This lesion was associated with older age and prevalent in both proximal and distal tube, but more common in the fimbrial end (12). The left vs. right location site of SCOUT did not correlate with the location of the primary serous cancer (12). Thus, this lesion may be a surrogate marker of HGSC.

p53 signature. The third lesion is p53 signature, which is defined as a linear expansion of $>12$ of morphologically normal epithelium with p53 overexpression (12). This lesion shares identical p53 mutations and other genomic changes with HGSC, but lacks excessive cell proliferation (16). p53 signature is seen predominantly in continuity with STIC, localizes to the same (fimbria) region as STIC and shares preneoplastic properties with HGSC including p53 mutations $(5,17)$. p53 signature is frequently identified in serous cancer $(66 \%)$ and may promote p53-driven preneoplastic transformation (12). However, controversy exists as to the incidence and clinical significance of p53 signature. This lesion is rare in non-serous cancer (18). Conversely, it can occasionally be found even in non-cancer patients (12).

STIC. The last is STIC, which is characterized by the presence of a discrete lesion, single or multiple, and located in the fimbriated end of the tube. STIC lesions display epithelial stratification and mitotic figures, demonstrating atypical histologic changes $(13,19)$. The epithelium of STIC showed strong p53 positivity and harbors clonal TP53 mutations (12). It has been suggested that STIC is found in 11-68\% of HGSC patients $(12,20,21)$ as well as up to $60 \%$ of sporadic cancer cases and in $0.6-10 \%$ of the carriers of patients with hereditary cancer (22). Conversely, the frequency of fallopian tube precursors in benign gynecologic diseases was approximately $20 \%$ of SCOUT, $10 \%$ of p53 signature and $<4 \%$ of STIC cases $(1,2)$. STIC was exclusively observed in patients with HGSC (3) and more common in the ipsilateral side of dominant HGSC (12). This alteration is considered as a precursor or an early event in the oncogenesis of HGSC. Thus, STIC may be a malignant lesion with metastatic potential to ovarian $\operatorname{HGSC}(21,23)$. Another possibility is that STIC is regarded as an intraepithelial metastasis from HGSC to the fallopian tube (24).

In the present study, we characterized histopathological alterations recognized currently in the fallopian tube epithelium, including a 'SCE-SCOUT-p53 signature-STIC-HGSC sequence' model (1-13). SCE and SCOUT are not considered to be preneoplastic lesions. SCOUT, p53 signatures, and STIC are frequently identified in HGSC. p53 signature is a low-grade preneoplastic condition, whereas STIC is considered as a true high-grade preneoplastic lesion, with a significant risk for HGSC development. HGSC may exhibit a continuous spectrum of a variety of lesions ranging from normal to precursor, premalignant, and finally malignant lesions.

\section{Molecular pathogenesis}

Differences in gene and protein expression between precursors and malignant lesions identify a continuous disease spectrum underlying HGSC. Investigators have characterized the stepwise changes of molecular profiles identified by genomic, proteomic and immunohistochemical approaches, including BCL2 (BCL2 apoptosis regulator), p73 (tumor protein p73), PAX2 (paired box 2), p53, PAX8 (paired box 8), H2AX (H2A histone family member X), STMN1 (stathmin 1), EZH2 (enhancer of zeste 2 polycomb repressive complex 2 subunit), Ki67, ALDH1A1 (aldehyde dehydrogenase 1 family member A1), CTNNB1 ( $\beta$-catenin), CCNE1 (cyclin E1), LAMC1 (Laminin $\gamma 1$ ), and HMGA2 (high mobility group AT-hook 2) genes and telomere shortening (Table I) $(3,5,12,13,16,25-37)$.

Normal fallopian epithelium. Non-ciliated cells, such as secretory cells, in normal fallopian tubal epithelium were PAX2-positive, but ciliated cells were PAX2-negative (25). The protein encoded by the $P A X 2$ gene induces epithelial invagination to form a tubular structure in Müllerian duct precursors of the coelomic epithelium. The fimbria is exposed to estrogen and progesterone in a cyclic manner. Estrogen can induce differentiation of fallopian epithelium to a ciliated 
Table I. Differences in protein expression between precursors and malignant lesions.

Fallopian tube epithylium

\begin{tabular}{|c|c|c|c|c|c|c|c|c|}
\hline Markers & Secretory cells & Ciliated cells & OSE & SCE & SCOUT & p53 signature & STIC & HGSC \\
\hline BCL2 & + & - & - & & + & + & + & + \\
\hline p73 & - & + & - & & - & & & \\
\hline PAX2 & + & - & $+/-$ & & - & - & - & - \\
\hline p53 & - & - & - & - & - & + & + & + \\
\hline PAX8 & + & - & $+/-$ & & & & + & + \\
\hline$\gamma \mathrm{H} 2 \mathrm{AX}$ & & & & - & - & & + & + \\
\hline STMN & - & & $+/-$ & & + & + & + & + \\
\hline EZH2 & & & & & + & & + & + \\
\hline ALDH1 & + & + & $+/-$ & & $+/-$ & - & - & - \\
\hline CCNE1 & & & & & & & + & + \\
\hline Telomere shortening & & & & & & & + & + \\
\hline Laminin $\gamma 1$ & & & & & & & + & + \\
\hline HMGA2 & - & $+/-$ & & & & & + & + \\
\hline
\end{tabular}

+, positive staining; -, negative staining; +/-, weak staining; OSE, ovarian surface epithelium; SCE, secretory cell expansion; SCOUT, secretory cell outgrowth; STIC, serous tubal intraepithelial carcinoma; HGSC, high-grade serous cancer.

phenotype (26). A member of the PAX family, PAX8, also plays a role in organogenesis of the Müllerian system and is a marker of fallopian tubal secretory cells and ovarian surface epithelium (OSE) $(16,27)$. Other molecular characteristics identified in fallopian tube epithelium are as follows: Fallopian tubal epithelium comprises different phenotypic and molecular subtypes; secretory cells (BCL2+, PAX2+, PAX8+, HMFG2+, p73-, FOXJ1-, and acetylated tubulin-) and ciliated cells (BCL2-, PAX2-, PAX8-, HMFG2-, p73+, FOXJ1+, LhS28+, acetylated tubulin+, and Sall2+) (16,25-27).p73 is a homologue of the p53 and induces cell cycle arrest, conferring its tumor suppressive activity. HMGA2 is associated with the epithelial-mesenchymal process. A monoclonal antibody, LhS28, reacts with basal bodies of ciliated epithelial cells. BCL2 gene encodes an integral outer mitochondrial membrane protein that blocks the apoptotic death. These specific molecules can function as promising markers to track a stepwise progression of fallopian tube epithelium to precursor lesions to carcinoma.

OSE/CIC. OSE did not exhibit tubal biomarkers, BCL2, p73, FOXJ1 and phospho-Smad2, while PAX8 was expressed by OSE (27). Steroid hormones convert mesothelial-derived OSE to Müllerian-type tissues including CIC (28). CIC is a heterogeneous group and can be divided into two types; ciliated (tubal-type, PAX8+ and p73 4 ) or flat (OSE-type, calretinin+) (28). CIC, but not OSE, had heterogeneous p73 staining with a ciliated phenotype. PAX8+ CIC undergoes Müllerian metaplasia.Furthermore, OSE specifically expressed STMN1 oncogene which regulates cytoskeletal dynamics, cell cycle progression, mitosis, and cell migration $(29,30)$. STMN1+ OSE possesses a highly proliferative potential. STMN1 upregulation was reported in highly proliferative breast cancers and in ovarian cancers. The PAX8+/STMN1+ OSE cells may promote a preneoplastic phenotype in these cysts participating to a pro-tumoral niche.
SCOUT. SCOUT has normal-appearing tubal epithelium without atypia (5). This lesion is identified by BCL2+, STMN1+, EZH2+, p53-, p73-, PAX2-, PAX8-, MIB1-, ALDH1A1-, and $\gamma$-H2AX- $(5,13,25,31)$. The majority of SCOUT is typically associated with wild-type p53 expression (do not overexpress p53) and a loss of PAX2 and p73 expression $(13,25)$. SCOUT may be a non-obligate precursor of HGSC. However, a small proportion of SCOUT is identified by p53+ and p73+ in fallopian tubes from women with inherited and sporadic HGSC (5). SCOUT, p53 signature, STIC and HGSC share a positive staining for STMN1 and EZH2 and negative staining for PAX2 (25). P53 signature, STIC and HGSC exhibit a negative staining for ALDH1A1 (31). STMN1 and EZH2 are cancer-associated genes and involved in cell cycle progression and proliferation.

p53 signature. In the p53 signature, abnormal p53 protein accumulation was observed by immunohistochemistry. The p53 signature contains an altered expression of multiple genes and pathways within histologically unremarkable precursor in benign tubal epithelium (13). This lesion was identified by $\mathrm{p} 53+$, BCL2+, STMN1+, PAX2- and a low Ki-67 proliferation index $(3,5,13)$. Overexpression of p53 protein (usually associated with mutation) may serve as a useful diagnostic marker in the assessment of HGSC and its precursor lesions, suggesting an essential role for p53 mutation in early serous tumorigenesis.

STIC. STIC exhibits a panel of immunohistochemical markers, TP53+, BCL2+, $\gamma-\mathrm{H} 2 \mathrm{AX}+, \mathrm{STMN} 1+, \mathrm{EZH} 2+$, PAX8+, Laminin $\gamma 1+$, HMGA2+, MIB1+, Ki67+, CCNE1+ and PAX2- $(3,12,25,32-37)$. The distribution of HMGA2 immunoreactivity overlapped with TP53 mutation-positive STIC (37). The finding of short telomeres and overexpression of CCNE1 in STIC may be the earliest molecular changes in 
chromosomal instability and carcinogenesis $(32,35,36)$. STIC may therefore be an immediate precursor of HGSC.

HGSC. Immunohistochemical studies demonstrated that HGSC exhibited p53+, PAX8+, BCL2+, HMGA2+, STMN1+, EZH2+, PAX2- and ALDH1A1- (3,12,25,27,29,31,33,34,37). Since PAX8 is frequently expressed by secretory cells of the normal fallopian tube and OSE, these cells are thought to be the origin of HGSC. ALDH1A1 was expressed in secretory and ciliated tubal epithelial cells and OSE, but was absent in p53 signature, STIC and HGSC (31). ALDH1A1 loss seems to be an early event in HGSC carcinogenesis, suggesting that ALDH1A1 may act as a tumor suppressor.

In this review, we have demonstrated detailed aspects of stepwise deterioration during HGSC progression from precursors to carcinoma. The immunostainings of p53, STMN1, EZH2, BCL2, CCNE1, Ki67 and $\gamma$-H2AX were significantly increased in a stepwise manner from SCOUT to p53 signature, STIC, and finally HGSC. Conversely, PAX2 and ALDH1A1 expression was decreased during the early phase of SCOUT development. The pathogenesis of HGSC may be centered on cell cycle deregulation, cell proliferation and anti-apoptosis. The current model of serous carcinogenesis can be stated as a set of two core predictions: HGSC is exemplified through stepwise cancer development with a particular sequence of molecular alterations, including PAX2, ALDH1A1, TP53, STMN1, EZH2 and BCL2; and the evolutionary trajectory of HGSC progression is rapid because secondarily acquired genetic alterations may occur independently.

\section{Conclusion}

Historically, HGSC was believed to originate from OSE cells that form CIC $(2,23,27)$. CIC did not present before menarche and the number of CIC increased with age (28). Coexisting cell hyperplasia and papillary growth were observed in OSE of ovaries from aged women (30). A greater lifetime number of ovulatory cycles, incessant ovulation, leads to localized OSE injury and inflammation, which increases ovarian cancer risk $(2,27,38)$. A previous study revealed that CIC correlates with low-grade serous and endometrioid tumors, but not HGSC (39). Descriptive evidence failed to show a direct link between morphologic changes and genomic alterations in HGSC arising from CIC foci. CIC may originate from implantation of tubal epithelium when the OSE is disrupted at ovulation (28).

The Müllerian-type tubal epithelium results in the formation of CIC by a process of implantation of tubal tissue rather than by a process of metaplasia from OSE with mesothelium-derived lining (40). Thus, more recent evidence supports the idea that most HGSC in both sporadic and hereditary ovarian cancer are of fallopian tubal origin $(23,41,42)$. Despite the fact that an obvious precursor STIC was contiguous with invasive carcinoma, one third of these patients were not associated with STIC in the fallopian tube (41). Fallopian tube hosts progenitor to the majority of HGSC. However, we were not able to deny a possibility that nearby OSE, CIC, and the tuboperitoneal junctional epithelium are all involved in pelvic serous carcinogenesis $(12,43)$. Therefore, not only the fallopian tubes, but also OSE, CIC and peritoneum can be linked to this malignancy.
Ongoing research is likely to identify molecular and genetic factors that are critical in the development of HGSC. A series of morphological changes from normal fallopian epithelium to preneoplastic to neoplastic lesions were concomitant with multistep accumulation of molecular and genetic alterations. Recent studies provide a stepwise progression of fallopian tubal epithelium to precursor lesions to carcinoma, with the aid of a 'secretory cell-SCE-SCOUT-p53 signature-STIC-HGSC sequence' model. Immunohistochemical markers, including p53, STMN1, EZH2, CCNE1, Ki67 and $\gamma-\mathrm{H} 2 \mathrm{AX}$, were gradually increased during the SCOUT-p53 signature-STIC-HGSC sequence. Conversely, PAX2 expression was decreased during early phase of SCOUT development.

In conclusion, the present review provides a descriptive molecular pathology in a serous carcinogenic sequence model. We summarize the current understanding of temporal and spatial changes of candidate markers in HGSC development.

\section{Acknowledgements}

The present study was supported by grant-in-aid for Scientific Research from the Ministry of Education, Science, and Culture of Japan to the Department of Obstetrics and Gynecology, Nara Medical University (to H.K.).

\section{References}

1. Nishida N, Murakami F and Higaki K: Detection of serous precursor lesions in resected fallopian tubes from patients with benign diseases and a relatively low risk for ovarian cancer. Pathol Int 66: 337-342, 2016.

2. Zeppernick F, Meinhold-Heerlein I and Shih IeM: Precursors of ovarian cancer in the fallopian tube: Serous tubal intraepithelial carcinoma - an update. J Obstet Gynaecol Res 41: 6-11, 2015.

3. Wen J, Shi JL, Shen DH, Chen YX and Song QJ: Morphologic changes of fallopian tubal epithelium in ovarian serous tumors. Zhonghua Bing Li Xue Za Zhi 41: 433-437, 2012 (In Chinese).

4. Li J, Ning Y, Abushahin N, Yuan Z, Wang Y, Wang Y, Yuan B Cragun JM, Chambers SK, Hatch K, et al: Secretory cell expansion with aging: Risk for pelvic serous carcinogenesis. Gynecol Oncol 131: 555-560, 2013.

5. Chen EY, Mehra K, Mehrad M, Ning G, Miron A, Mutter GL, Monte N, Quade BJ, McKeon FD, Yassin Y, et al: Secretory cell outgrowth, PAX2 and serous carcinogenesis in the Fallopian tube. J Pathol 222: 110-116, 2010.

6. Carlson JW, Miron A, Jarboe EA, Parast MM, Hirsch MS, Lee Y, Muto MG, Kindelberger D and Crum CP: Serous tubal intraepithelial carcinoma: Its potential role in primary peritoneal serous carcinoma and serous cancer prevention. J Clin Oncol 26: 4160-4165, 2008

7. Carcangiu ML, Radice P, Manoukian S, Spatti G, Gobbo M, Pensotti V, Crucianelli R and Pasini B: Atypical epithelial proliferation in fallopian tubes in prophylactic salpingo-oophorectomy specimens from BRCA1 and BRCA2 germline mutation carriers. Int J Gynecol Pathol 23: 35-40, 2004.

8. Leunen K, Legius E, Moerman P, Amant F, Neven P and Vergote I: Prophylactic salpingo-oophorectomy in 51 women with familial breast-ovarian cancer: Importance of fallopian tube dysplasia. Int J Gynecol Cancer 16: 183-188, 2006.

9. Piek JM, van Diest PJ, Zweemer RP, Jansen JW, Poort-Keesom RJ, Menko FH, Gille JJ, Jongsma AP, Pals G, Kenemans P and Verheijen RH.: Dysplastic changes in prophylactically removed Fallopian tubes of women predisposed to developing ovarian cancer. J Pathol 195: 451-456, 2001.

10. Crum CP, Drapkin R, Miron A, Ince TA, Muto M, Kindelberger DW and Lee Y: The distal fallopian tube: A new model for pelvic serous carcinogenesis. Curr Opin Obstet Gynecol 19: $3-9,2007$.

11. Jarboe EA, Folkins AK, Drapkin R, Ince TA, Agoston ES and Crum CP: Tubal and ovarian pathways to pelvic epithelial cancer: A pathological perspective. Histopathology 53: 127-138, 2008. 
12. Mittal N, Srinivasan R, Gupta N, Rajwanshi A, Nijhawan R, Gautam U, Sood S and Dhaliwal L: Secretory cell outgrowths, p53 signatures, and serous tubal intraepithelial carcinoma in the fallopian tubes of patients with sporadic pelvic serous carcinoma. Indian J Pathol Microbiol 59: 481-488, 2016.

13. Li HX, Lu ZH, Shen K, Cheng WJ, Malpica A, Zhang J, Wei JJ, Zhang ZH and Liu J: Advances in serous tubal intraepithelial carcinoma: correlation with high grade serous carcinoma and ovarian carcinogenesis. Int J Clin Exp Pathol 7: 848-857, 2014.

14. Ebihara T, Yoshimura Y, Shiraki M, Maruyama K, Ichikawa F, Kawakami S, Fukushima M and Oda T: Role of endosalpinx in the oviductal environment. Nihon Sanka Fujinka Gakkai Zasshi 41: 881-887, 1989 (In Japanese).

15. Piek JM, van Diest PJ, Verheijen RH and Kenemans P: Cell cycle-related proteins p21 and bcl-2: Markers of differentiation in the human fallopian tube. Histopathology 38: 481-482, 2001.

16. Yamamoto Y, Ning G, Howitt BE, Mehra K, Wu L, Wang X, Hong Y, Kern F, Wei TS, Zhang T, et al: In vitro and in vivo correlates of physiological and neoplastic human Fallopian tube stem cells. J Pathol 238: 519-530, 2016.

17. Huang HS, Hsu CF, Chu SC, Chen PC, Ding DC, Chang MY and Chu TY: Haemoglobin in pelvic fluid rescues Fallopian tube epithelial cells from reactive oxygen species stress and apoptosis. J Pathol 240: 484-494, 2016.

18. Vang R, Visvanathan K, Gross A, Maambo E, Gupta M, Kuhn E, Li RF, Ronnett BM, Seidman JD, Yemelyanova A, et al: Validation of an algorithm for the diagnosis of serous tubal intraepithelial carcinoma. Int J Gynecol Pathol 31: 243-253, 2012.

19. Weinberger V, Bednarikova M, Cibula D and Zikan M: Serous tubal intraepithelial carcinoma (STIC) - clinical impact and management. Expert Rev Anticancer Ther 16: 1311-1321, 2016.

20. Chen F, Gaitskell K, Garcia MJ, Albukhari A, Tsaltas J and Ahmed AA: Serous tubal intraepithelial carcinomas associated with high-grade serous ovarian carcinomas: A systematic review. BJOG 124: 872-878, 2017.

21. Schneider S, Heikaus S, Harter P, Heitz F, Grimm C, Ataseven B, Prader S, Kurzeder C, Ebel T, Traut A and du Bois A: Serous tubal intraepithelial carcinoma associated with extraovarian metastases. Int J Gynecol Cancer 27: 444-451, 2017.

22. Malmberg K, Klynning C, Flöter-Rådestad A and Carlson JW: Serous tubal intraepithelial carcinoma, chronic fallopian tube injury, and serous carcinoma development. Virchows Arch 468 707-713, 2016.

23. Corzo C, Iniesta MD, Patrono MG, Lu KH and Ramirez PT: Role of fallopian tubes in the development of ovarian cancer. J Minim Invasive Gynecol 24: 230-234, 2017.

24. Eckert MA, Pan S, Hernandez KM, Loth RM, Andrade J, Volchenboum SL, Faber P, Montag A, Lastra R, Peter ME, et al: Genomics of ovarian cancer progression reveals diverse metastatic trajectories including intraepithelial metastasis to the fallopian tube. Cancer Discov 6: 1342-1351, 2016.

25. Ning G, Bijron JG, Yamamoto Y, Wang X, Howitt BE, Herfs M, Yang E, Hong Y, Cornille M, Wu L, et al: The PAX2-null immunophenotype defines multiple lineages with common expression signatures in benign and neoplastic oviductal epithelium. J Pathol 234: 478-487, 2014.

26. Comer MT, Leese HJ and Southgate J: Induction of a differentiated ciliated cell phenotype in primary cultures of Fallopian tube epithelium. Hum Reprod 13: 3114-3120, 1998

27. Adler E, Mhawech-Fauceglia P, Gayther SA and Lawrenson K: PAX8 expression in ovarian surface epithelial cells. Hum Pathol 46: 948-956, 2015.
28. Banet $\mathrm{N}$ and Kurman RJ: Two types of ovarian cortical inclusion cysts: Proposed origin and possible role in ovarian serous carcinogenesis. Int J Gynecol Pathol 34: 3-8, 2015.

29. Karst AM, Levanon K, Duraisamy S, Liu JF, Hirsch MS, Hecht JL and Drapkin R: Stathmin 1, a marker of PI3K pathway activation and regulator of microtubule dynamics, is expressed in early pelvic serous carcinomas. Gynecol Oncol 123: 5-12, 2011.

30. Bajwa P, Nagendra PB, Nielsen S, Sahoo SS, Bielanowicz A, Lombard JM, Wilkinson JE, Miller RA and Tanwar PS: Age related increase in mTOR activity contributes to the pathological changes in ovarian surface epithelium. Oncotarget 7: 19214-19227, 2016

31. Chui MH, Wang Y, Wu RC, Seidman J, Kurman RJ, Wang TL and Shih IeM: Loss of ALDH1A1 expression is an early event in the pathogenesis of ovarian high-grade serous carcinoma. Mod Pathol 28: 437-445, 2015.

32. Kuhn E, Wang TL, Doberstein K, Bahadirli-Talbott A, Ayhan A, Sehdev AS, Drapkin R, Kurman RJ and Shih IeM: CCNE1 amplification and centrosome number abnormality in serous tubal intraepithelial carcinoma: Further evidence supporting its role as a precursor of ovarian high-grade serous carcinoma. Mod Pathol 29: 1254-1261, 2016

33. Novak M, Lester J, Karst AM, Parkash V, Hirsch MS, Crum CP, Karlan BY and Drapkin R: Stathmin 1 and p16 (INK4A) are sensitive adjunct biomarkers for serous tubal intraepithelial carcinoma. Gynecol Oncol 139: 104-111, 2015.

34. Kuhn E, Kurman RJ, Soslow RA, Han G, Sehdev AS, Morin PJ, Wang TL and Shih IeM: The diagnostic and biological implications of laminin expression in serous tubal intraepithelial carcinoma. Am J Surg Pathol 36: 1826-1834, 2012.

35. Kuhn E, Meeker A, Wang TL, Sehdev AS, Kurman RJ and Shih IeM: Shortened telomeres in serous tubal intraepithelial carcinoma: An early event in ovarian high-grade serous carcinogenesis. Am J Surg Pathol 34: 829-836, 2010

36. Sehdev AS, Kurman RJ, Kuhn E and Shih IeM: Serous tubal intraepithelial carcinoma upregulates markers associated with high-grade serous carcinomas including Rsf-1 (HBXAP), cyclin E and fatty acid synthase. Mod Pathol 23: 844-855, 2010.

37. Wei JJ, Wu J, Luan C, Yeldandi A, Lee P, Keh P and Liu J: HMGA2: A potential biomarker complement to P53 for detection of early-stage high-grade papillary serous carcinoma in fallopian tubes. Am J Surg Pathol 34: 18-26, 2010.

38. Fathalla MF: Incessant ovulation - -a factor in ovarian neoplasia? Lancet 2: 163, 1971.

39. Merritt MA and Cramer DW: Molecular pathogenesis of endometrial and ovarian cancer. Cancer Biomark 9: 287-305, 2010.

40. Kurman RJ: Origin and molecular pathogenesis of ovarian high-grade serous carcinoma. Ann Oncol 24: x16-x21, 2013.

41. Meserve EEK, Brouwer J and Crum CP: Serous tubal intraepithelial neoplasia: The concept and its application. Mod Pathol 30: 710-721, 2017.

42. Gilks CB, Irving J, Köbel M, Lee C, Singh N, Wilkinson N and McCluggage WG: Incidental nonuterine high-grade serous carcinomas arise in the fallopian tube in most cases: Further evidence for the tubal origin of high-grade serous carcinomas. Am J Surg Pathol 39: 357-364, 2015.

43. Crum CP, Herfs M, Ning G, Bijron JG, Howitt BE, Jimenez CA, Hanamornroongruang S, McKeon FD and Xian W: Through the glass darkly: Intraepithelial neoplasia, top-down differentiation, and the road to ovarian cancer. J Pathol 231: 402-412, 2013. 